\title{
La comunicación digital de las ONG españolas de apoyo al Sáhara
}

\section{Digital communication of Spanish NGOs in support of Western Sahara}
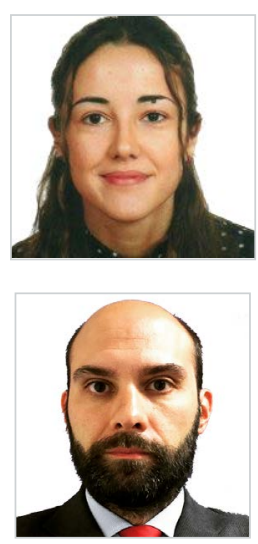

Laura de Cos Carrera. Doctoranda en Comunicación Audiovisual y Publicidad, Licenciada en Publicidad y Relaciones Públicas por la Universidad Complutense de Madrid y cofundadora de la asociación solidaria con el pueblo saharaui Nuestra Jaima. Sus líneas de investigación están relacionadas con la Comunicación en las organizaciones sin ánimo de lucro.

Universidad Complutense de Madrid, España

Idecos@ucm.es

ORCID: 0000-0002-3963-6685

Luis Mañas Viniegra. Doctor en Comunicación Audiovisual y Publicidad. Profesor asociado en las Universidades Complutense de Madrid y Rey Juan Carlos de Madrid y miembro del Grupo de Investigación Complutense Gestión de las Marcas y Procesos de Comunicación. Sus principales líneas de investigación son la gestión de intangibles en la marca corporativa y la aplicación de las nuevas tecnologías a la innovación docente. Su última publicación inicia uno de los temas en los que se centrarán sus próximas investigaciones: Mañas-Viniegra, L. (2017). "Storytelling digital en la comunicación corporativa: Comparativa entre IBEX 35 y Dow Jones". El Profesional de la información, v. 26, n. 6, pp. 1127-1138.

Universidad Complutense de Madrid, España

Imanas@ucm.es

ORCID: 0000-0001-9129-5673

Recibido: 06/11/2017 - Aceptado: 05/12/2017

\section{Resumen:}

Esta investigación analiza cuantitativa y cualitativamente las acciones de comunicación que realizan las ONG españolas de apoyo al Sáhara, así como su nivel de profesionalización. Se realizó un estudio exploratorio basado en un análisis de contenido de las webs de las entidades miembro de CEAS, realizando posteriormente entrevistas semiestructuradas a sus responsables para profundizar en la gestión de su comunicación. Como conclusión, se observa un escaso nivel de profesionalización y la necesidad de incorporar a profesionales de la comunicación en redes sociales.

\section{Palabras clave:}

Comunicación; redes sociales; ONG; Sáhara; web 2.0.
Received: 06/11/2017 - Accepted: 05/12/2017

\section{Abstract:}

This research analyses quantitatively and qualitatively the communicative actions carried out by Spanish NGOs in support of Western Sahara, as well as their level of professionalism. An exploratory study was conducted based on content analysis of the websites of members of CEAS (State Coordinating Committee of Solidarity with Western Sahara Associations), and then subsequently carrying out semi-structured interviews with their directors to delve deeper into the management of these NGOs' communications. In conclusion, it has been observed that the level of professionalism is low, and there is a need to incorporate communication specialists in social media.

\section{Keywords:}

Communication; social media; NGO; western Sahara; web 2.0.

Cómo citar este artículo:

De Cos Carrera L. y Mañas Viniegra, L. (2018). La comunicación digital de las ONG españolas de apoyo al Sáhara. Doxa Comunicación, 26, 13-34. 


\section{Introducción}

El Sáhara Occidental fue territorio español entre 1884 y 1976. En un contexto de descolonización del continente africano, España se comprometió a celebrar en 1975 un referéndum para decidir el futuro del Sáhara Occidental con el auspicio de la ONU (Martín Beristain y González Hidalgo, 2012: 49). Este hecho originó un conflicto con las pretensiones anexionistas de Marruecos que concluyó con la firma de los Acuerdos de Madrid, entregando la parte norte del Sáhara Occidental a Marruecos y la parte sur, a Mauritania. El 27 de febrero de 1976, los saharauis proclaman la República Árabe Saharaui Democrática y se inicia entonces un conflicto bélico que enfrenta a las tres partes durante doce años y que provoca la huida de la mayoría de la población civil saharaui hacia el desierto de Argelia, donde se construyeron los campos de refugiados de Tinduf. En 1979 Mauritania decidió poner fin a la ocupación de la parte del Sáhara que le correspondía, pero inmediatamente fue invadida por Marruecos, quien reivindicaba su derecho legítimo, defendiendo el territorio con la construcción de ocho muros defensivos en el desierto para evitar la vuelta de los refugiados saharauis al territorio (El Observador, 2012: 40).

En 1988 se inició un proceso de paz aceptado por ambas partes y liderado por la ONU y la Organización para la Unidad Africana (OUA), que se firmó en 1991, incluyendo un alto el fuego, el despliegue en la zona del Consejo de Seguridad de la ONU y un periodo de transición hacia el referéndum de autodeterminación del Sáhara, de cuyo resultado dependería la independencia o su integración en Marruecos. A pesar de que la fecha máxima prevista para el referéndum era el año 1992, las discrepancias sobre el origen saharaui de algunos integrantes del censo (Entrialgo, 2011: 16) llevaron a un estancamiento de las negociaciones. Actualmente, el Sáhara Occidental es un territorio no autónomo pendiente de descolonización (Um Draiga, 2013) y la ONU no reconoce la soberanía ejercida por Marruecos (ONU, 2006: 11), calificada como una ocupación (Soroeta Liceras, 2005) que configura la última colonia existente en África (de Currea Lugo, 2011).

Mientras tanto, la población saharaui vive en la zona ocupada por Marruecos, donde se ha denunciado una vulneración sistemática de los Derechos Humanos (Amnistía Internacional, 2016: 11), o en los campamentos de refugiados, donde casi 165.000 personas (ACNUR, 2016) dependen de la ayuda humanitaria internacional y continúan esperando una solución.

Los medios de comunicación tienen gran capacidad para determinar qué asuntos poseen interés informativo (Shaw, 1979) y, como no ha existido ningún avance significativo desde hace décadas, la mediatización de la situación del pueblo saharaui ha decaído con el paso de los años. Consecuentemente, nos encontramos en un contexto en el que muchas personas, especialmente los jóvenes, desconocen la existencia del conflicto del Sáhara Occidental, a pesar de que en España existen numerosas Organizaciones No Gubernamentales (ONG) que fomentan su difusión. Por ello, esta investigación analiza la gestión de la comunicación de las ONG españolas de apoyo al Sáhara.

\subsection{La comunicación digital de las ONG españolas}

Las ONG se crean con el objetivo de impulsar la cooperación para el desarrollo a partir de la concienciación de los públicos y/o la puesta en marcha de proyectos (Latorre Tapis, 2001: 103), sin olvidar la importancia de dinamizar y fomentar los procesos sociales de cambio y la participación ciudadana (Marí Sáez, 2016: 155). Para lograrlo, estas organizaciones, incluidas en el denominado tercer sector, deben concebir la comunicación como una función estratégica (Arroyo Almaraz, 2012: 1; 
Balas Lara, 2008: 17) y considerarla como un elemento transversal capaz de sensibilizar, concienciar y generar confianza y reputación como agentes de transformación social (Martín Nieto, 2007: 132; Balas Lara, 2008: 32).

"La comunicación es una parte esencial de estas organizaciones porque está ligada a su propia razón de existir. Así, por ejemplo, mientras las empresas comunican para existir (vender más), las entidades sin ánimo de lucro existen para comunicar (por una sociedad mejor)" (Vidal y Creixams, 2006: 1).

En efecto, las ONG encuentran su razón de ser en la transmisión de determinados valores a la sociedad, que contribuyen a hacerla más democrática, habitable y humana, pero "el reto es hacerlo cada vez con mayor profesionalidad, creatividad y eficacia" (Vidal Climent, 2004: 322), a pesar de que la gestión de la comunicación sea aún una de sus principales carencias (Herranz de la Casa, 2010: 108). Esta situación es especialmente preocupante en las ONG con menores recursos, con gran dificultad para darse a conocer, aparecer en los medios y proyectarse en el entorno:

"Carecemos de una estrategia de comunicación social adecuada. Tenemos poca capacidad para trasmitir a la sociedad problemas muy importantes, la gente no nos conoce o nos conoce mal, a menudo no conseguimos captar su interés. [...] La comunicación social es una asignatura pendiente en la mayor parte de las asociaciones" (Fundación Esplai, 2002: 20).

Resulta paradójica, a pesar de estos fines sociales, la visión instrumental y mediática de la comunicación que tienen muchas de estas organizaciones, orientándola hacia la búsqueda de financiación (Bernabé, 2001: 145; Balas Lara, 2011: 197). En este escenario, un mayor uso de las nuevas tecnologías fomentaría un cambio en su comunicación hacia la creación de mejores contenidos y una mayor sensibilización y participación de los públicos (Arroyo Almaraz y Martín Nieto, 2011: 46; Arroyo Almaraz, Baladrón Pazos y Martín Nieto, 2013: 85), aprovechando una mayor difusión basada en la comunicación directa de un modo flexible y sin elevados costes (Martín Nieto, 2007: 135; Herranz de la Casa, 2010: 204). La comunicación a través de las herramientas digitales posibilita que ésta sea más efectiva, transparente, participativa, plural, inclusiva y horizontal (Montoliu y Riu, 2012: 11).

Prácticamente todas las ONG utilizan actualmente una página web para presentarse, dar a conocer su trabajo y aumentar su visibilidad, pero son pocas las que aprovechan la interactividad, la inmediatez y los nuevos modos de participación que ofrece Internet (Arroyo Almaraz y Martín Nieto, 2011: 250; Fundación Un Sól Món, 2002: 22). En este sentido, el uso de redes sociales como instrumento de comunicación en las ONG responde a la necesidad de este tipo de organizaciones de emplear "tecnologías digitales de bajo coste (webs, redes sociales, blogs, etc.) que facilitan las tareas de información, participación u organización en red" (Cárcar Benito, 2015: 136; Lema Blanco, Rodríguez Gómez y Barranquero Carretero, 2016: 92) con el objetivo de obtener una adecuada difusión (Couldry, 2010). De este modo, públicos tan diversos como los socios, mecenas, voluntarios y los propios medios de comunicación encuentran en la comunicación online de las ONG un espacio en el que informarse, interactuar e integrar un proyecto solidario del que son partícipes (Soria Ibáñez, 2011: 177).

Sin embargo, estos objetivos difícilmente pueden cumplirse si la comunicación digital de las ONG carece de una auténtica interactividad (Soria Ibáñez, 2011: 184) que facilite la participación ciudadana y la propia bidireccionalidad de la información, características esenciales de la web 2.0 (O’Reilly, 2005). Precisamente, la máxima expresión de esta bidireccionalidad, orientada hacia el cambio social la pueden alcanzar las ONG a través de las redes sociales, puesto que "favorecen las prescripciones realizadas libremente por los usuarios y con ellas se facilita la viralidad y el engagement personal" (Arroyo 
Almaraz y Baños González, 2013: 333). El uso de las redes sociales esté especialmente indicado, puesto que "permiten una transmisión de valores” (Baraybar Fernández, 2009: 42) y ofrecen a los usuarios una participación activa que va más allá de la información que reciben (García Galera y del Hoyo Hurtado, 2013).

La tardía profesionalización de las ONG en los años 80 (González Luis, 2006: 32), de sus departamentos de comunicación a partir de los años 90-2000 (Sampedro, Jerez Novara y López Rey, 2002: 264; Soria Ibáñez, 2010: 289), la escasez de personas contratadas en plantilla (Lema Blanco, Rodríguez Gómez y Barranquero Carretero, 2016: 97) e, incluso, la falta de apoyo de los estamentos directivos (Arroyo Almaraz, Baladrón Pazos y Martín Nieto, 2013: 85) limitan el uso de las redes sociales para la consecución de la movilización social y los mensajes difundidos son meramente informativos y tienen una "limitada capacidad para captar la atención de los públicos a los que se dirigen” (Arroyo Almaraz y Baños González, 2013: 351). Existe, en cualquier caso, una polarización entre el uso reactivo de las de escaso presupuesto y el proactivo de las grandes organizaciones sociales (Cammaerts, 2005), impulsadas hasta 2008 por un ciclo de crecimiento económico y una considerable inversión pública (Bahamonde Silva, García Mirón y Martínez Rolán, 2017: 439).

La gestión de la comunicación es una función estratégica dentro de las organizaciones, por lo que éstas ya "no demandan exclusivamente profesionales de la comunicación sino profesionales de los activos intangibles por medio de la comunicación" (Carrillo Durán, 2016: 273). En un contexto de constante digitalización, las organizaciones deben contar con la figura profesional del community manager como gestor de comunidades online en las que inicia y monitoriza conversaciones con sus públicos, involucrándolos en los fines de la organización y gestionando la reputación online (Marquina Arenas, 2012). El perfil de los community managers en las agencias españolas de publicidad y relaciones públicas es el de "una mujer de entre 25 y 35 años, que ha estudiado Periodismo y cuenta con una experiencia previa de 2 ó 3 años en medios digitales; que además considera imprescindible tener una formación específica en comunicación” (Silva Robles, 2016: 243).

Las ONG de acción social, las de mayor tamaño, están presentes en Facebook (96\%), Twitter (92\%) y Youtube (80\%) de forma mayoritaria, a pesar de que "la información facilitada a los socios, donantes o colaboradores y la relación con éstos revela cierta desidia” (Bahamonde Silva, García Mirón y Martínez Rolán, 2017: 444). Sin embargo, la falta de profesionalización y de personal especializado en las ONG de menor tamaño implica un grave problema en la gestión de las redes sociales en la medida en que la inmediatez, es decir, una actualización permanente de los mensajes en redes sociales, otorga mayor credibilidad a la fuente entre sus públicos (Waterman, Spence y Van de Heide, 2013).

Las investigaciones realizadas (Waters et al., 2009; Lim, 2012) han demostrado los beneficios que Facebook proporciona a las ONG en la relación e interacción con sus públicos, a los que invita a "movilizarse y adherirse a sus reivindicaciones" (Soria Ibáñez, 2010: 297), destacando "un importante porcentaje de jóvenes que participan en las redes con propósitos solidarios o cívicos" (García Galera, del Hoyo Hurtado y Fernández Muñoz, 2014: 41). A pesar de ello, la medición de la eficacia de los mensajes en redes sociales tampoco es una actividad prioritaria en las ONG (Arroyo Almaraz, Baladrón Pazos y Martín Nieto, 2013: 85) y éstas miden el impacto de sus páginas web de forma poco sistemática y rigurosa, si es que lo hacen (Fundación Un Sól Món, 2002: 40).

"En ocasiones, resulta paradójico pensar que muchas personas opinan y creen en la importancia de la comunicación y, sin embargo, la realidad contrasta con los escasos recursos que se invierten para potenciarla” (Herranz de la Casa, 2010: 109). 
Todas las carencias puestas de manifiesto repercuten directamente en la labor realizada por las ONG de apoyo al Sáhara, que consideran que entre las causas del silencio en los medios de comunicación españoles respecto al conflicto saharaui se encuentran la antigüedad de un conflicto que pierde interés frente a otros, la falta de sensibilidad de algunos corresponsales para ponerlo de manifiesto en sus medios o la ausencia de interés por parte de los propios ciudadanos en el tema (Diario de Mallorca, 2008). Las propias asociaciones reconocen que el programa de acogida de niños en verano ha ido decayendo con los años y España ha pasado de acoger a 10.000 niños anualmente a principios del siglo XXI a 4.500 en 2014 y 3.000 en 2017 (El Confidencial Saharaui, 2017). Por tanto, es imprescindible mejorar la gestión de la comunicación en el sector, que debe ser coherente con cada organización y los recursos con los que cuenta, tanto económicos como profesionales.

\section{Métodos}

El presente artículo investiga la gestión de la comunicación de las ONG de apoyo al Sáhara en España y su grado de profesionalización. El hecho de que la causa saharaui haya ido cayendo en el olvido con el paso de los años y la falta de profesionalización en comunicación de las organizaciones constituyen las principales causas que justifican la idoneidad de realizar este estudio.

El objetivo general de esta investigación es analizar las acciones de comunicación que realizan las ONG solidarias con el pueblo saharaui, así como el nivel de profesionalización de dicha comunicación.

Los objetivos específicos son los siguientes:

- Situar la comunicación de la causa saharaui.

- Describir los objetivos de comunicación que persiguen las ONG en la comunicación de la causa saharaui.

- Conocer el uso que las ONG que apoyan al Sáhara hacen de la web y las redes sociales y la importancia que otorgan a la comunicación online.

- Conocer el nivel de profesionalización de la comunicación en las organizaciones, identificar posibles causas y descubrir las posibilidades que tienen de mejorar y maximizar la eficacia de sus mensajes.

- Obtener resultados sobre la comunicación que realizan las asociaciones y hacer recomendaciones, donde fuera posible, sobre mejoras en la comunicación.

La hipótesis de partida es que las ONG de apoyo al Sáhara están poco profesionalizadas en el ámbito de la comunicación.

La metodología utilizada tras la revisión bibliográfica del estado de la cuestión ha sido el análisis de contenido de las páginas web y redes sociales de las organizaciones que se ocupan de la causa saharaui, que se ha realizado en febrero de 2017. Para ello, se ha realizado previamente un censo de las diferentes organizaciones de solidaridad con el Sáhara existentes en nuestro país a partir de la página web de la Coordinadora Estatal de Asociaciones Solidarias con el Sáhara (CEAS Sáhara), donde se muestran las 18 entidades federadas a la misma. De las 18 asociaciones miembro, descartamos profundizar en diez de ellas por alguna de las siguientes razones: 
- No poseen página web.

- Los contenidos de la página no coinciden con la asociación.

- La página proporciona escasa información sobre la organización y sus objetivos, los contenidos se limitan a noticias o actividades muy concretas de índole regional, no poseen redes sociales, etc.

En cualquier caso, dichas asociaciones no reúnen los requisitos mínimos para poder estudiar su comunicación. Por tanto, quedan las ocho asociaciones restantes y la Coordinadora, es decir, nueve organizaciones a analizar:

- CEAS Sáhara - Coordinadora Estatal de Asociaciones Solidarias con el Sáhara.

- FANDAS - Federación Andaluza de Asociaciones Solidarias con el Sáhara.

- Um Draiga - Amigos del Pueblo Saharaui en Aragón.

- AAPSIB - Asociación de Amigos del Pueblo Saharaui de las Islas Baleares.

- Cantabria por el Sáhara.

- FEMAS - Federación de las Asociaciones de Amistad con el Pueblo Saharaui en la Comunidad de Madrid.

- SOGAPS - Solidariedade Galega co Pobo Saharaui.

- ANARASD - Asociación Navarra de Amigos y Amigas de la República Árabe Saharaui Democrática.

- Asociación de Amigos y Amigas de la RASD de Álava.

Posteriormente, se han realizado entrevistas semiestructuradas a los responsables de cada ONG en su calidad de expertos para profundizar en la gestión de su comunicación, que han estado centradas en conocer la comunicación de las organizaciones y la profesionalización de las mismas. El contenido de las entrevistas se ha analizado a partir de los ejes discursivos que establecen líneas temáticas dentro del análisis discursivo (Benavides Delgado, 2005). De las nueve asociaciones contactadas, no obtuvimos respuesta de dos de ellas: AAPSIB (Islas Baleares) y FANDAS (Andalucía).

\section{Resultados}

\subsection{Análisis de contenido de las páginas web}

El 100\% de las entidades ofrecen información en sus páginas web sobre quiénes son, sus objetivos y un método de contacto. El $66,67 \%$ cuenta con un apartado destinado a formas de colaboración y el $88,89 \%$ tiene uno sobre la agenda de sus actividades, aunque la mayoría sin actualizar o en desuso. Llama la atención que dos de las asociaciones $(22,22 \%)$, a pesar de tener redes sociales, no incluyan su acceso desde la página. Otras, aunque lo hacen, se muestra de forma poco visible, por lo que hay que buscar exhaustivamente el enlace para encontrarlo. 
Laura de Cos Carrera y Luis Mañas Viniegra

Cuadro 1. La comunicación de las ONG a través de sus páginas web

\begin{tabular}{|c|c|c|c|c|c|c|c|c|c|}
\hline ONG & $\begin{array}{l}\text { Página } \\
\text { web }\end{array}$ & $\begin{array}{l}\text { Quiénes } \\
\text { somos }\end{array}$ & Objetivos & $\begin{array}{l}\text { Cómo } \\
\text { colaborar }\end{array}$ & Contacto & Agenda & Prensa & $\begin{array}{c}\text { Redes } \\
\text { sociales }\end{array}$ & Total (\%) \\
\hline CEAS Sáhara & Sí & Sí & Sí & Sí & Sí & Sí & Sí & Sí & 100,00 \\
\hline FANDAS & Sí & Sí & Sí & No & Sí & Sí & Sí & Sí & 87,50 \\
\hline Um Draiga & Sí & Sí & Sí & No & Sí & Sí & Sí & Sí & 87,50 \\
\hline AAPSIB & Sí & Sí & Sí & Sí & Sí & No & No & Sí & 75,00 \\
\hline Cantabria por el Sáhara & Sí & Sí & Sí & Sí & Sí & Sí & Sí & No & 87,50 \\
\hline FEMAS & Sí & Sí & Sí & No & Sí & Sí & Sí & No & 75,00 \\
\hline FEDESAEX & Sí & Sí & No & No & Sí & Sí & No & No & 50,00 \\
\hline SOGAPS & Sí & Sí & Sí & Sí & Sí & Sí & Sí & Sí & 100,00 \\
\hline ANARASD & Sí & Sí & Sí & Sí & Sí & Sí & No & Sí & 87,50 \\
\hline Asociación Álava & Sí & Sí & Sí & Sí & Sí & Sí & Sí & Sí & 100,00 \\
\hline ACAPS (Cataluña) & Sí & Sí & Sí & No & Sí & No & No & No & 50,00 \\
\hline Asociación Asturiana & Sí & Sí & No & No & Sí & No & No & No & 37,50 \\
\hline ASOCSPS & No & No & No & No & No & No & No & No & 0,00 \\
\hline ACAPS (Canarias) & Sí & No & No & No & Sí & No & No & No & 25,00 \\
\hline Federación Valencia & Sí & No & No & No & No & No & No & No & 12,50 \\
\hline Asociación Riojana & No & No & No & No & No & No & No & No & 0,00 \\
\hline ANAS & Sí & Sí & Sí & No & Sí & No & No & No & 50,00 \\
\hline UACYLSPS & No & No & No & No & No & No & No & No & 0,00 \\
\hline Delegación Castilla-La Mancha & No & No & No & No & No & No & No & No & 0,00 \\
\hline Total (\%) & 78,95 & 68,42 & 57,89 & 31,58 & 73,68 & 47,37 & 36,84 & 36,84 & \\
\hline
\end{tabular}

Fuente: elaboración propia

\subsubsection{Objetivos de las organizaciones}

Tras examinar los objetivos que establecen las organizaciones en sus respectivas páginas web, podemos concluir que todas siguen la misma línea y persiguen las mismas metas:

- El reconocimiento de la República Árabe Saharaui Democrática (RASD).

- Trabajar para que el Pueblo Saharaui pueda ejercer su derecho de libre autodeterminación e independencia cultural, económica, política y social.

- Desarrollar proyectos de cooperación al desarrollo y ayuda humanitaria para los campamentos de población refugiada. 
- Denunciar la situación en el Sáhara Occidental.

- Dar a conocer la causa saharaui entre la población. Sensibilizar, concienciar mediante charlas, coloquios, exposiciones fotográficas y otras actividades.

\subsection{Análisis de contenido de las redes sociales}

El 77,78\% de las asociaciones tienen una página en Facebook ${ }^{1}$, el 44,44\%, una cuenta en Twittery sólo el 22,22\%, en YouTu$b e$. De estos datos se desprende que Facebook comprende el grueso de las comunicaciones relacionadas con el Sáhara en redes sociales por parte de las organizaciones, ya que es ahí donde se encuentran principalmente. Asimismo, se ha comprobado que las cuentas estén actualmente activas. Las asociaciones que las utilizan suelen publicar con frecuencia y todas ellas muestran un registro de actividad reciente, por lo que efectivamente hacen uso de las mismas.

Cuadro 2. Redes sociales que poseen las $\mathrm{ONG}^{2}$

\begin{tabular}{|l|c|c|c|}
\hline \multicolumn{1}{|c|}{ ONG } & Facebook & Twitter & YouTube \\
\hline CEAS Sáhara & Sí & Sí & No \\
\hline FANDAS & Sí & No (desuso) & No \\
\hline Um Draiga & No (perfil) & Sí & No \\
\hline AAPSIB & Sí & No & Sí \\
\hline Cantabria por el Sáhara & Sí & No & No \\
\hline FEMAS & Sí & No & No \\
\hline SOGAPS & Sí & Sí & No \\
\hline ANARASD & No (perfil) & No & No \\
\hline Asociación Álava & Sí & Sí & Sí \\
\hline \multicolumn{1}{r|}{ Total (\%) } & $\mathbf{7 7 , 7 8}$ & $\mathbf{4 4 , 4 4}$ & $\mathbf{2 2 , 2 2}$ \\
\hline
\end{tabular}

Fuente: elaboración propia.

Tras analizar todas las publicaciones en Facebook (257 en total) de las diferentes asociaciones que tienen página en la red social durante el mes de febrero de 2017, obtenemos que el 74,71\% de las mismas son noticias relacionadas con el Sáhara o contenido ajeno compartido en la página. El 14,01\% cuenta con contenido propio -o externo, pero aportando algo nuevoy sólo el 11,28\% de las publicaciones hacen referencia a actividades desarrolladas por las ONG. Respecto a la frecuencia de publicación de contenidos, de media se publica cada tres días y todas ellas publican, como mínimo, una vez por semana.

1 El criterio para determinar que una asociación utiliza la red social Facebook es que tenga una página, no siendo contabilizado un perfil.

2 El criterio del “sí” aplica cuando la organización posee cuenta en la red social y la utiliza. El criterio entre paréntesis del “no" especifica si la organización tiene cuenta, pero lleva mucho tiempo en desuso o no está orientada al público (ej.: tienen perfil, pero no página). 
Laura de Cos Carrera y Luis Mañas Viniegra

Cuadro 3. Análisis de publicaciones en Facebook durante febrero de 2017

\begin{tabular}{|l|c|c|c|c|c|}
\hline \multicolumn{1}{|c|}{ ONG } & $\begin{array}{c}\text { Contenido ajeno } \\
\text { compartido }\end{array}$ & Actividades & $\begin{array}{c}\text { Contenido propio/ajeno } \\
\text { con aportación }\end{array}$ & $\begin{array}{c}\text { Total } \\
\text { publicaciones }\end{array}$ & $\begin{array}{c}\text { Frecuencia media } \\
\text { de publicación }\end{array}$ \\
\hline CEAS Sáhara & 2 & 2 & 6 & 10 & 3,7 días \\
\hline FANDAS & 5 & 1 & 4 & 10 & 3,3 días \\
\hline AAPSIB & 15 & 2 & 5 & 22 & 1,7 días \\
\hline Cantabria por el Sáhara & 156 & 8 & 7 & 171 & Todos los días \\
\hline FEMAS & 7 & 4 & 4 & 15 & 2,7 días \\
\hline SOGAPS & 7 & 8 & 10 & 25 & 2 días \\
\hline Asociación Álava & - & 4 & - & 4 & 7 días \\
\hline Total (\%) & $\mathbf{7 4 , 7 1}$ & $\mathbf{1 1 , 2 8}$ & $\mathbf{1 4 , 0 1}$ & &
\end{tabular}

Fuente: elaboración propia.

\subsection{Acciones de comunicación de las ONG del Sáhara}

Los datos iniciales de cada asociación nos muestran las diferencias que hay entre ellas en el número de personas contratadas, en los socios y los voluntarios, en las vías de financiación y, finalmente, en el presupuesto destinado a la comunicación.

Cuadro 4. Personas, vías de financiación y presupuesto de comunicación en las ONG

\begin{tabular}{|l|c|c|c|l|c|}
\hline \multicolumn{1}{|c|}{ ONG } & Contratados & Socios & Voluntarios & \multicolumn{1}{c|}{ Vías financiación } & Presupuesto comunicación \\
\hline CEAS Sáhara & 3 & $\mathrm{No}^{3}$ & $\mathrm{No}^{3}$ & Cuotas entidades miembro & 0 \\
\hline Um Draiga & 0 & 180 & 25 & $\begin{array}{l}\text { Admon. Pública: } 90 \%, \\
\text { cuota socios: 5\%, } \\
\text { actividades y venta: } 5 \%\end{array}$ & 5.820 \\
\hline Cantabria por el Sáhara & 1 & 191 & 15 & Cuotas de socios y actividades & 500 \\
\hline FEMAS & 0 & 15 & Todos & Cuotas de socios & Depende del año \\
\hline SOGAPS & 4 & 136 & 70 & Organismos oficiales & Escaso \\
\hline ANARASD & 3 & 110 & 30 & No responde & Marginal \\
\hline Asociación Álava & 5 & 201 & 35 & Fondos públicos 95\% y privados 5\% & \\
\hline
\end{tabular}

Fuente: elaboración propia.

3 No hay socios ni voluntarios individuales, son las entidades miembro 9-11. 


\subsubsection{Comunicación}

El 28,57\% de las organizaciones (Um Draiga y SOGAPS) afirman tener un departamento específico para la comunicación, mientras que el 42,86\% niega contar con él y el 28,57\% restante no responde a la pregunta en el cuestionario. Habrá que tener en cuenta la profesionalización de dicho departamento en las asociaciones que lo tengan y la formación de las personas que trabajen en él.

El 71,43\% de las asociaciones no tiene ninguna persona contratada para encargarse de la comunicación. Solo dos de las siete asociaciones que respondieron al cuestionario tienen una persona encargada de realizar las tareas de este ámbito: una de ellas (Asociación Álava) es una profesional de la información (Grado en Periodismo) y la otra no tiene formación en comunicación (SOGAPS).

Ninguna asociación cuenta con un plan de comunicación publicado en su página web para que los diferentes públicos puedan conocerlo. El 14,29\% (una ONG) afirma tener un plan de comunicación, pero no tenerlo a disposición del público (SOGAPS) y el 71,43\% no tiene un plan de comunicación establecido. La asociación restante (Álava) incluye la comunicación dentro de un Plan Estratégico de Educación para la transformación social.

\section{Cuadro 5. Asociaciones con un Plan de Comunicación establecido} Sí, está publicado para que lo conozcan los
diferentes públicos

Sí, pero no está publicado

\section{Otro}

No

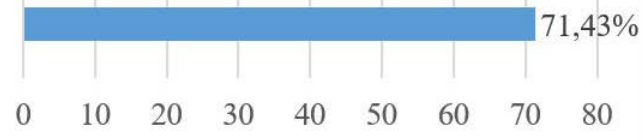

Fuente: elaboración propia.

El 100\% de las asociaciones afirman dirigirse a la sociedad en general, el 57,14\% a los públicos internos y el mismo porcentaje, a los medios de comunicación. El 42,86\% se dirige a la Administración y el 14,29\%, a otras ONG.

Queda patente que el público principal de estas asociaciones es la sociedad civil, donde pueden hallar simpatizantes, socios, voluntarios... Sin embargo, llama la atención que no haya más entidades que se dirijan a la Administración y a los medios de comunicación, ya que tienen una clara posición respecto al conflicto saharaui y luchan por conseguir un cambio para el cual estos públicos pueden jugar un papel fundamental. 
El 71,43\% declara ajustar sus mensajes a los diferentes públicos a los que se dirigen, frente al 28,57\% restante, que no lo hace. Habría que indagar en cada uno de esos públicos, puesto que la sociedad abarca un amplio número de públicos distintos.

Aunque para el 57,14\% de las asociaciones Internet es una herramienta imprescindible para desarrollar su trabajo y comunicarlo, consideramos que el 28,57\% que opina que Internet es una herramienta más (FEMAS y ANARASD), sin mayor importancia que cualquier otra, es un número muy elevado teniendo en cuenta que hoy el 80,6\% de la población utiliza Internet (INE, 2016) y la oportunidad que supone la red para este tipo de entidades. Estos datos pueden ser resultado de la falta de personal especializado en la comunicación que tenga un conocimiento suficiente sobre el tema.

Cuadro 6. Papel de Internet dentro de las asociaciones

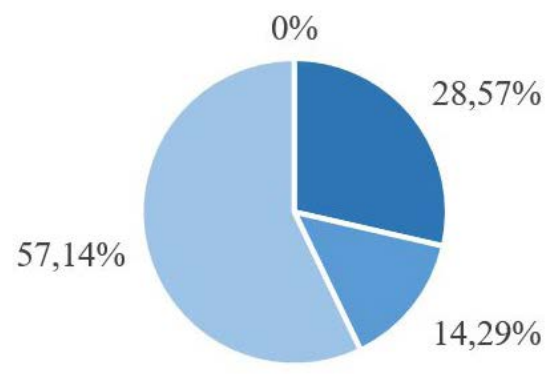

- No es útil

- Una herramienta más

- Importante

- Imprescindible

Fuente: elaboración propia.

\subsubsection{Página web}

Todas las ONG menos una, que no respondió (85,71\%), afirman haber tenido en cuenta elementos de diseño gráfico a la hora de elaborar su página web. El 42,86\% de páginas fueron diseñadas por un profesional del diseño gráfico ( $14,29 \%$ contratados y $28,57 \%$ voluntarios).

Respecto a las características que poseen las páginas web desde su punto de vista, la más destacada es la sencillez de la misma, marcada por un 85,71\% de las ONG. Cabe mencionar que SOGAPS, la asociación de Galicia, marcó todas las características, en contraste con ANARASD, la de Navarra, que no marcó ninguna.

A pesar de que el $85,71 \%$ de las asociaciones afirmaban haber tenido en cuenta criterios de diseño gráfico al crear la web, sólo el 28,57\% señaló que la página estuviese bien diseñada (SOGAPS y la asociación de Álava). Asimismo, únicamente una asociación utilizó la opción de intuitiva para designar su web, una característica importante a la hora de facilitar la navegación al usuario y aumentar el tiempo de visita. 
Cuadro 7. Características de las páginas web según las propias asociaciones

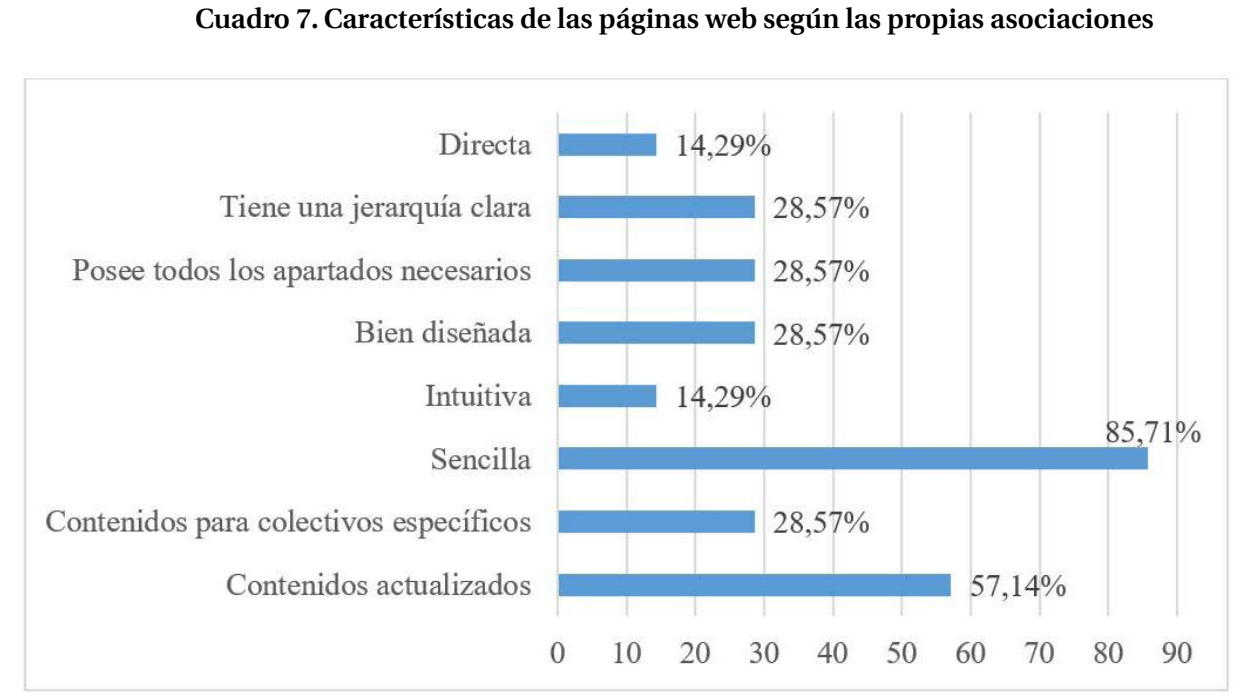

Fuente: elaboración propia.

El 71,43\% de las personas encargadas del mantenimiento de la página web no son especialistas en ello; sólo una organización (Um Draiga; 14,29\%) dice tener un encargado profesional de la web (voluntario). Una de las asociaciones expuso que actualmente no había nadie que se encargase de la página.

La mayoría (71,43\%) no analizan el tráfico de su web ni realizan estudios sobre sus usuarios. Por tanto, al no haber ninguna herramienta de control del tráfico, es difícil saber de dónde proceden las visitas, qué contenidos son más populares, las palabras clave con mejores resultados, etc. Um Draiga y SOGAPS fueron quienes indicaron que sí lo analizan.

\subsubsection{Redes sociales}

Para identificar las posibilidades que atribuyen las asociaciones a las redes sociales, así como las barreras, miedos o inconvenientes que encuentran en su utilización, establecimos unas preguntas abiertas para conocer de primera mano su visión. El análisis del discurso proporciona una reiteración en determinadas respuestas:

Posibilidades:

- Llegar a un público más amplio, de forma directa, inmediata (rápida), sencilla y sin costes.

- Permiten interaccionar con los diferentes públicos y facilitan la comunicación.

- Difundir su trabajo y la causa por la que trabajan.

- Barreras, miedos, inconvenientes:

- Hay que reaccionar muy rápido y requieren de mucho tiempo. 
- Otros frenos mencionados sólo por una asociación cada uno:

- Comentarios imprudentes, faltas de respeto.

- Riesgos de hackeo, boicots, etc.

- Cada vez es más necesaria la inversión económica, porque las redes favorecen el contenido pagado y ocultan el orgánico.

- Ninguno, siempre que se utilicen adecuadamente.

La red social más utilizada es Facebook (71,43\%) ${ }^{4}$, seguida de Twitter, con un 57,14\%. Sólo una ONG tiene cuenta en YouTube y una de las asociaciones no utiliza ninguna red social. Estos resultados confirman los obtenidos en el análisis de datos de contenido, aunque las cifras no sean las mismas, ya que no todas las ONG respondieron al cuestionario.

Cuadro 8. Redes sociales de las asociaciones

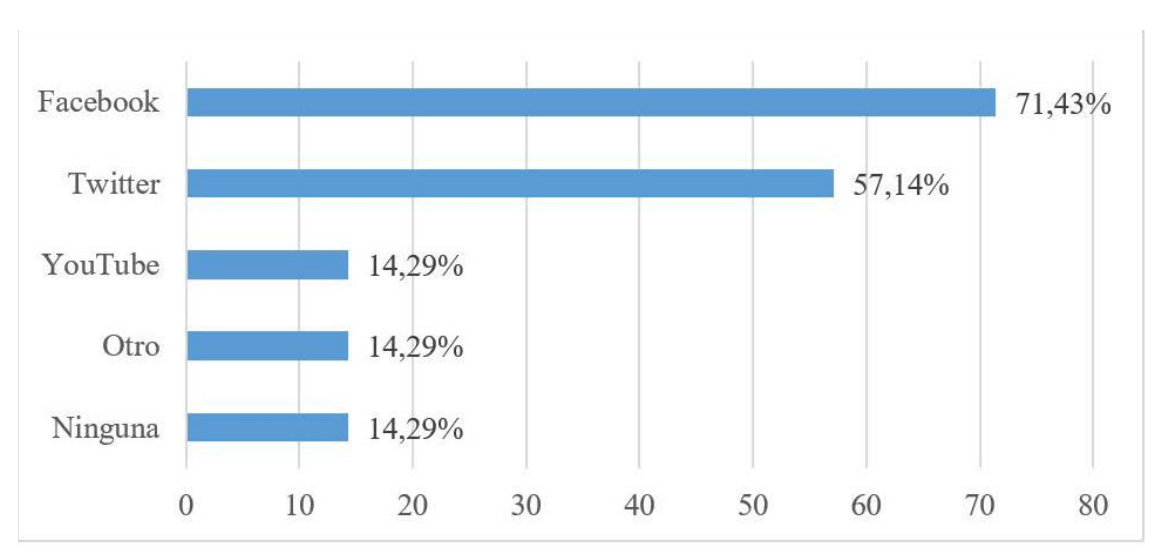

Fuente: elaboración propia.

Todas las ONG que tienen cuenta en las redes sociales, que como hemos comprobado son principalmente Facebook y Twitter, las utilizan y actualizan con frecuencia, como mencionamos en el análisis de contenidos: el $33,33 \%$ diariamente, el $33,33 \%$ cada dos o tres días y el 33,33\% restante al menos una vez a la semana.

Ninguna de las asociaciones tiene un community manager o una persona formada para dicho cargo. En dos de ellas (33,33\%), los encargados de actualizar las redes sociales son los responsables de comunicación contratados (SOGAPS y Álava) y, en otra (16,67\%), esta función la realiza una persona contratada, pero no responsable de comunicación. En el $50 \%$ restante, son los propios voluntarios de la organización quienes se encargan de actualizar las redes sociales.

4 El 100\% de las asociaciones afirmaron que tenían Facebook. Sin embargo, como se aprecia en el análisis de datos de contenido, dos de ellas tienen un perfil y no una página, por lo que no figurarán como usuarias de esta red social. 
Todas las asociaciones que tienen una página de Facebook excepto una que no respondió, es decir, el 66,67\% de las mismas, miden la eficacia de sus mensajes en las redes sociales. El principal indicador para medir dicha incidencia es el número de veces que se ha compartido (80\%), seguido del número de seguidores, los comentarios y el alcance de las publicaciones (60\%). En tercer lugar, encontramos las reacciones a las publicaciones y el tráfico a la web, con un $40 \%$ de asociaciones que lo tienen en cuenta, y el número de clics a las publicaciones (20\%). De nuevo, SOGAPS fue la única en marcar todas las opciones.

Llama la atención que solo dos ONG (40\%) afirmen utilizar las reacciones (likes, etc.) de los usuarios para observar la eficacia de sus mensajes en redes sociales, ya que suele ser lo primero que se ve en las propias publicaciones. Además, podemos ver cómo parece que prestan más atención al alcance (60\%) que a las reacciones, cuando lo primero indica a cuántas personas les ha salido o han visto la publicación, mientras que las reacciones o los clics en las publicaciones (20\%) infieren cierto interés, por lo que se estaría anteponiendo el número de personas a las que llega nuestro contenido frente al número de personas que se interesa por él, aunque es cierto que la interacción de compartir también refleja el atractivo de las publicaciones y que cuantas más veces se comparten, mayor es el alcance.

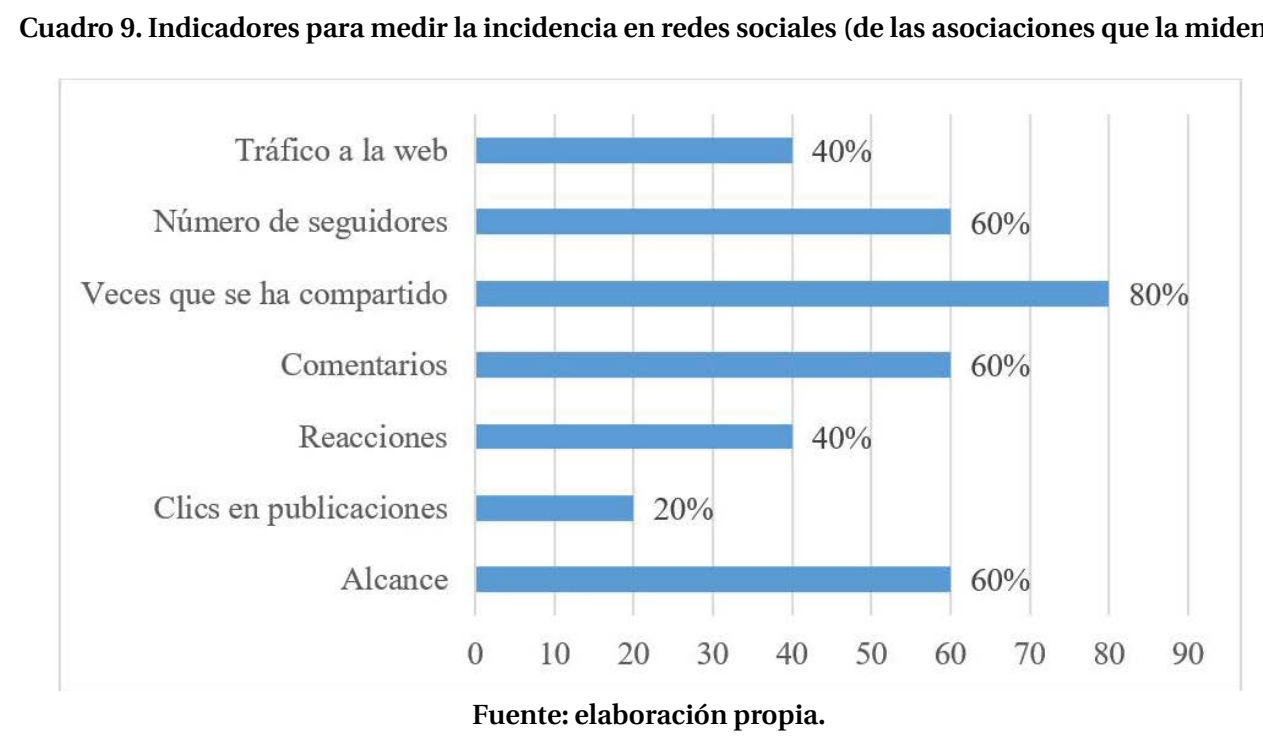

\subsubsection{Publicidad}

En lo que respecta a la publicidad (prensa, televisión, radio, Internet...), se muestra una clara falta de acciones, debido principalmente a la falta de presupuesto. Todas las asociaciones, excepto una (Asociación Álava) que afirma que no haber contemplado la opción de hacer publicidad porque "no va con su filosofía", consideran que no tienen presupuesto sufi- 
ciente, por lo que toda la publicidad que hacen, si es que la hacen, es en espacios gratuitos, con independencia del medio. Sólo encontramos una pequeña excepción, SOGAPS, que afirma que también realiza publicidad pagada en prensa.

\subsubsection{Relaciones públicas}

Todas las asociaciones realizan ruedas de prensa, gestión de entrevistas, envío de notas de prensa, eventos, etc. con el fin de obtener notoriedad o aparecer en los medios de comunicación. Sin embargo, la mayoría de ellas (71,43\%) no miden la eficacia de estas acciones. Las dos asociaciones que sí miden la eficacia son Cantabria por el Sáhara y la Asociación de Álava.

Más de la mitad de las asociaciones $(57,14 \%)$ no realizan colaboraciones de patrocinio con otras entidades o empresas para financiar sus actividades o una parte de ellas.

\subsection{Autopercepción de las debilidades y fortalezas}

El estudio realizado finaliza con el diagnóstico de las debilidades y fortalezas detectadas en la comunicación a partir de la autopercepción de las propias asociaciones.

En cuanto a los puntos fuertes, encontramos una gran variedad de respuestas, desde cualidades propias de la comunicación de la asociación (página web actualizada y con diseño, posibilidad de colaborar a través de la web...), hasta una asociación que reconoce no tener puntos fuertes. También hubo respuestas que atribuían la fortaleza comunicativa de la entidad a agentes externos (personas que colaboran con la difusión) o al reconocimiento que tienen en su trayectoria. Asimismo, vemos respuestas que a nuestro juicio no constituyen un punto fuerte, sino algo a mejorar: Cantabria por el Sáhara considera que la comunicación de su página de Facebook es muy buena porque se actualiza diariamente. Sin embargo, de media tiene más de 100 publicaciones al mes, lo que supone una saturación de la misma.

Por otra parte, parece que los puntos débiles están más claros que las fortalezas, ya que las respuestas son más concisas y directas. Algunos de los puntos a mejorar, según las asociaciones, serían los siguientes:

- Elaborar contenidos propios.

- Medir la eficacia del esfuerzo realizado.

- Personal dedicado a la comunicación.

- Actualización de la página web.

- Comunicación dirigida al público joven.

- Crear una estrategia y un Plan de Comunicación.

- Mejorar la visibilidad en las redes sociales.

- Um Draiga pone de manifiesto la necesidad de coordinación entre las organizaciones y su escasa capacidad para generar contenidos actualmente:

- "Debería existir una coordinación mayor entre todas las webs del Sáhara para no repetir y centrarnos cada uno en un aspecto concreto del conflicto".

- "En la parte técnica estamos todas las webs en un nivel bajo, la mayoría repetimos lo que ya está publicado". 


\section{Discusión}

A grandes rasgos, se aprecia una amplia diferencia entre lo que las asociaciones hacen y lo que consideran que se debería hacer. Hemos visto que son una minoría las ONG que cuentan con un departamento de comunicación (28,57\%) que tienen una persona contratada para estas tareas (28,57\%), disponen de un plan de comunicación (14,29\%) o de un encargado de la web especialista (14,29\%). Ninguna tiene un community manager o una persona formada para dicho cargo concreto, sólo en torno al $14 \%$ de las publicaciones de Facebook tiene contenido propio y el $11 \%$ muestra las actividades desarrolladas. El 28,57\% analiza el tráfico y los usuarios de su página y ese mismo porcentaje mide los resultados de las notas y ruedas de prensa, entrevistas, etc. realizadas.

Por otro lado, para el 57,14\%, Internet es una herramienta imprescindible. El 71,43\% declara ajustar sus mensajes a los diferentes públicos, el 66,67\% de las asociaciones con cuenta en Facebook mide la eficacia de sus mensajes y el 85,71\% ha tenido en cuenta elementos de diseño gráfico al elaborar su página web.

Aunque hay aspectos en los que es palpable el esfuerzo que hacen estas organizaciones por realizar una comunicación adecuada, es cierto que se entrevé una falta de profesionalización, con independencia de los motivos causales.

En base a los datos recogidos a lo largo de esta investigación, se ha podido comprobar que gran parte de las tareas las realizan voluntarios, incluso hay asociaciones en las que todas las personas que trabajan en ellas lo hacen de manera altruista y compaginándolo con sus empleos. Por tanto, entendemos que la mayoría hace lo que puede con los recursos que tiene a su disposición y los conocimientos que poseen los involucrados.

Muchas de las deficiencias encontradas en la comunicación de las ONG son reconocidas por las mismas: falta de contenidos propios, ausencia de medición de resultados, falta de personal especializado, la escasa comunicación dirigida a los jóvenes, inexistencia de una estrategia y un plan de comunicación... Por tanto, las asociaciones son conscientes, en la mayoría de las ocasiones, de sus carencias y dificultades, pero no saben cómo mejorar en ese sentido.

A pesar de que las nuevas tecnologías, especialmente las redes sociales, ofrecen a las ONG ventajas como la gratuidad de su uso o el número de personas susceptible de interesarse por su contenido, no se aprovechan lo suficiente.

Aunque hay diferencias entre las asociaciones, ya que unas cuidan más la web, otras las redes sociales, las acciones offline y actividades, etc., y pese a que hay demasiado margen de mejora, en una valoración global de todos los aspectos planteados en la investigación, son SOGAPS y la Asociación de Amigos y Amigas de la RASD de Álava las ONG que exponen mayor constancia en los esfuerzos comunicativos, especialmente en los relacionados con las nuevas tecnologías. Estas dos asociaciones son quienes tienen una persona contratada para encargarse de la comunicación (siendo una de ellas una profesional de la materia y otra no), así como una estrategia para la comunicación, bien sea recogida en un plan de comunicación o en un plan estratégico que englobe este tema.

Un mayor rendimiento y número de visitas es consecuencia de contar con más personas contratadas, mayor presupuesto destinado a la comunicación y más tiempo invertido (Martín Nieto, 2007: 135). El hecho de que una persona esté contratada específicamente para estas tareas implica necesariamente un mayor número de horas dedicadas y, consecuentemente, 
mejores resultados. A pesar de ello, un mayor presupuesto no implica mejor rendimiento, ya que éste debe ir en consonancia con la profesionalidad. Al comienzo del análisis se apreció que ciertas organizaciones no destinaban un presupuesto a la comunicación, mientras que otras sí lo hacían y, sin embargo, a lo largo de la investigación se ha constatado que hay asociaciones que trabajan mejor a pesar de no tener o tener menor presupuesto.

Con un profesional de la comunicación dentro de la organización, no existirían aún asociaciones que desconozcan las posibilidades que les ofrece la red, ni que se muestren reticentes a su uso. También es importante destacar que una de las principales barreras que encontraron las asociaciones en la gestión de redes sociales es que requieren mucho tiempo y capacidad de reacción, algo que la contratación de un community manager resolvería, puesto que incluye una tarea más dentro de la gestión de la comunicación. De cara a maximizar la eficacia de los mensajes, habría que marcar qué es lo que queremos transmitir y qué es lo que le interesa a nuestro público, pensando de antemano a quién nos dirigimos, por qué y a través de qué canales. Se trata de adaptar el mensaje a nuestro público objetivo para intentar llegar a él de la forma más eficaz posible.

\section{Conclusiones}

A lo largo de esta investigación se han analizado las acciones de comunicación de las diferentes ONG españolas de apoyo al Sáhara, extrayendo las siguientes conclusiones:

1. Diez de las 18 entidades miembro de CEAS Sáhara no reúnen los requisitos mínimos para poder estudiar su comunicación.

2. Sólo una asociación (14,29\%) dispone de un profesional de la comunicación contratado para realizar tales funciones.

3. Hay un escaso nivel de profesionalización en la comunicación de las asociaciones solidarias con el Sáhara en España y existe la necesidad de incorporar a profesionales del sector.

4. Internet es una herramienta imprescindible para el $57,14 \%$ de las ONG analizadas.

5. Las asociaciones no aprovechan las posibilidades que les ofrecen las nuevas tecnologías. Es necesario que saquen mayor partido a estas herramientas, posean una página web propia bien diseñada y utilicen mejor las redes sociales para explotar todo su potencial.

6. Es indispensable que las organizaciones definan los objetivos y estrategias a seguir para poder establecer acciones en consonancia con su misión y su visión.

Específicamente, se ofrecen las siguientes recomendaciones a las ONG analizadas:

1. Aunar los esfuerzos de comunicación de las diferentes ONG agrupándose a través de CEAS Sáhara, pudiendo concretar cada una sus propios mensajes. Podría plantearse la posibilidad de crear una estrategia y un plan común, para lo cual haría falta invertir más recursos, tanto profesionales como económicos, pero a largo plazo se obtendrían mejores resultados. 
2. A pesar de que las asociaciones no se dedican únicamente a sensibilizar a la población, sino que cumplen con muchas otras labores (humanitarias, políticas, etc.) en lo que al Sáhara respecta, es necesaria una estrategia común, aunque se implante de forma individual.

3. Hay que considerar realizar una campaña viral a través de las redes sociales entre todas las asociaciones de España, desde las locales a las autonómicas, con la colaboración de alguna agencia y profesionales del sector, para alcanzar repercusión en los medios de comunicación, lo que permitiría llegar a un público más amplio y mejorar el conocimiento del tema por parte de la ciudadanía.

Como futura línea de investigación, en la que ya se está trabajando, se aplicarán estas conclusiones a una campaña de comunicación creada específicamente para una ONG cuyo objeto social es la ayuda al pueblo saharaui. De este modo, se podrá comprobar que las conclusiones obtenidas son extrapolables a cualquiera de las ONG que comparten el objeto de estudio.

\section{Referencias bibliográficas}

AAPSIB (s.f.): "Página web corporativa”. Disponible en: http://saharaillesbalears.org [Consultado el 10/03/2017].

ACAPS Canarias (s.f.): "Página web corporativa”. Disponible en: http://tenerifeconelsahara.blogspot.com.es/ [Consultado el 26/02/2017].

ACAPS Cataluña (s.f.): "Página web corporativa”. Disponible en: http://www.acaps.cat/ [Consultado el 26/02/2017].

ACNUR Comité Español (2016): “Refugiados saharauis: 40 años de vida en los campos”. Disponible en: http://bit.ly/2qpak6G [Consultado el 15/02/2017].

Amnistía Internacional (2016): “Informe 2015-2016. La situación de los Derechos Humanos en el mundo”. Disponible en: http://bit.ly/2s6vU05 [Consultado el 17/02/2017].

ANARASD (s.f.): "Página web corporativa”. Disponible en: http://www.anarasd.org/ [Consultado el 10/03/2017].

ANAS (s.f.): "Página web corporativa". Disponible en: http://sahara-navarra-sahara.blogspot.com.es/ [Consultado el 26/02/2017].

Arroyo Almaraz, I. y Baños González, M. (2013): “Tendencias de la comunicación del tercer sector en la web 2.0: Análisis retórico de los tropos". Icono 14, v. 11, n. 2, pp. 331-356. Disponible en: https://doi.org/10.7195/ril4.v11i2.556 [Consultado el 05/12/2017].

Arroyo Almaraz, I.; Baladrón Pazos, A. J. y Martín Nieto, R. (2013): “La comunicación en redes sociales: percepciones y usos de las Ong españolas". Cuadernos.info, n. 32, pp. 77-88. Disponible en: http://bit.ly/2rriGPq [Consultado el 27/02/2017].

Arroyo Almaraz, I. (2012): “La comunicación social eficiente del Tercer Sector”. Telos, n. 93, pp. 1-3. Disponible en: http:// bit.ly/2s6nExk [Consultado el 03/03/2017]. 
Arroyo Almaraz, I. y Martín Nieto, R. (2011): “La utilización de Internet en la comunicación expresiva de las ONG: Estudio exploratorio comparativo entre Argentina y España”. Zer. Revista de Estudios de Comunicación, vol. 16, n. 31, pp. $243-263$. Disponible en: http://bit.ly/1LCfq4R [Consultado el 25/02/2017].

Asociación Asturiana (s.f.): "Página web corporativa”. http://aaasahara.blogspot.com.es/ [Consultado el 26/02/2017].

Asociación de Amigos y Amigas de la RASD (s.f.): “Página web corporativa”. Disponible en: http://www.saharaelkartea.org/ es/ [Consultado el 10/03/2017].

Bahamonde Silva, X.; García Mirón, S. y Martínez Rolán, X. (2017): “Solidaridad y transparencia digital. Webs y redes sociales de las ONGs españolas de acción social”. El Profesional de la Información, vol. 26, n. 3, pp. 438-446. Disponible en: https:// doi.org/10.3145/epi.2017.may.10 [Consultado el 05/12/2017].

Balas Lara, M. (2011): La gestión de la comunicación en el Tercer Sector. Madrid: ESIC.

- (2008): "El reto de la comunicación del tercer sector". Revista Española del Tercer Sector, n. 8, pp. 17-37. Disponible en: http://bit.ly/22kc2mS [Consultado el 10/05/2017].

Baraybar Fernández, A. (2009): “Conectando valores: Las nuevas estrategias de la comunicación online en el Tercer Sector”. Icono 14, n. 13, pp. 37-55. Disponible en: https://doi.org/10.7195/ri14.v7i2.316 [Consultado el 05/12/2017].

Benavides Delgado, J. (2005): “Nuevas propuestas para el análisis del lenguaje en los medios”. Questiones publicitarias, vol. 1, n. 10, pp. 13-33.

Bernabé, J. (2001): “Las áreas de comunicación de las ONGD y el trabajo periodístico”. Revista Española de Desarrollo y Cooperación, n. 7, pp. 127-145.

Cammaerts, B. (2005): "ICT-usage among transnational social movements in the networked society: To organise, to mobilise and to debate", en Silverstone, R. (ed.): Media, technology and everyday life in Europe: from information to communication. Aldershot: Ashgate, pp. 73-92. Disponible en: http://eprints.lse.ac.uk/3278/ [Consultado el 05/12/2017].

Cantabria por el Sáhara (s.f.): "Página web corporativa”. Disponible en: http:/ / www.cantabriaporelsahara.com/ [Consultado el 10/03/2017].

Cárcar Benito, J. E. (2015): “Las redes y los movimientos sociales ¿una acción colectiva o marketing viral?” Icono 14, v. 13, n. 1, pp. 125-150. Disponible en: https://doi.org/10.7195/ri14.v13i1.744 [Consultado el 05/12/2017].

Carrillo Durán, M. V. (2016): “Importancia del profesional de la información en la dirección de comunicación de las organizaciones”. El Profesional de la Información, vol. 25, n. 2, pp. 272-278. Disponible en: https://doi.org/10.3145/ epi.2016.mar.14 [Consultado el 05/12/2017].

CEAS Sáhara (s.f.): "Página web corporativa”. Disponible en: http://ceas-sahara.es/ [Consultado el 10/03/2017].

Couldry, N. (2010): Why voice matters: Culture and politics after neoliberalism. London: Sage.

De Currea Lugo, V. (2011): “Sáhara Occidental: la última de las colonias de África”. Revista Análisis Internacional, n. 3, pp. 107-117. Disponible en: http://bit.ly/2qtHDEY [Consultado el 13/05/2017]. 
Diario de Mallorca (2008, 15 de febrero): "Periodistas especializados en el Sáhara denuncian el silencio de los medios de comunicación”. Diario de Mallorca. Disponible en: http://bit.ly/2rr5Kcl [Consultado el 14/05/2017].

El Confidencial Saharaui (2017, 11 de abril): "Vacaciones en Paz 2017 es el año con menor número de familias de acogida de la historia del proyecto”. El Confidencial Saharaui. Disponible en: http://bit.ly/2s0nZT5 [Consultado el 14/05/2017].

El Observador (2012): “El muro de la vergüenza marroquí”. El Observador, n. 52, pp. 40-42. Disponible en: http://bit. ly/1v3i7av. [Consultado el 14/02/2017].

Entrialgo, M. (2011): El conflicto del Sáhara en menos de 3.000 palabras. Oviedo: Coordinadora de ONGD del Principado de Asturias - Conseyu de la Mocedá del Principáu D’Asturies. Disponible en: http://bit.ly/2s0xdyE [Consultado el 10/02/2017]. FANDAS (s.f.): "Página web corporativa”. Disponible en: http://www.saharandalucia.org/ [Consultado el 10/03/2017].

Federación Valencia (s.f.): "Página web corporativa”. Disponible en: http://amicsaharapaisvalencia.blogspot.com.es/ [Consultado el 26/02/2017].

FEDESAEX (s.f.): "Página web corporativa”. Disponible en: https://www.federacionsaharaextremadura.org/ [Consultado el 26/02/2017].

FEMAS (s.f.): "Página web corporativa”. Disponible en: http://www.madridconelsahara.org/ [Consultado el 10/03/2017].

Fundación Esplai (2002): El Tercer Sector visto desde dentro. La renovación de las ONGs y los retos de la exclusión social. Barcelona: Fundación Esplai.

Fundación un Sól Món (2002): ¿Conectadas? Las ONG españolas en la red. Barcelona: Fundación un Sól Món. Disponible en: http://bit.ly/2qpyfD2 [Consultado el 12/05/2017].

García Galera, M. C.; del Hoyo Hurtado, M. y Fernández Muñoz, C. (2014): “Jóvenes comprometidos en la red: El papel de las redes sociales en la participación social activa”. Comunicar, vol. 22, n. 43, pp. 35-43. Disponible en: https://doi. org/10.3916/C43-2014-03 [Consultado el 05/12/2017].

García Galera, M. C. y del Hoyo Hurtado, M. (2013): “Redes sociales, un medio para la movilización juvenil”. Zer, v. 18, n. 34, pp. 111-125. Disponible en: http://www.ehu.eus/zer/hemeroteca/pdfs/zer34-06-garcia.pdf [Consultado el 05/12/2017].

González Luis, H. (2006): Estrategias de comunicación en las ONG de desarrollo. Departamentos, funciones e impacto en los medios. Madrid: CIDEAL. Disponible en: https://doi.org/10.13140/2.1.1694.6560 [Consultado el 05/12/2017].

Herranz de la Casa, J. M. (2010): La comunicación y la transparencia en las organizaciones no lucrativas. Madrid: Universidad Complutense de Madrid. Disponible en: http://bit.ly/2qm0LJH [Consultado el 14/03/2017].

Latorre Tapis, X. (2001): "El marketing aplicado a las ONGD: Coherencias e incoherencias en relación con la educación para el desarrollo". Comunicar, vol. 9, n. 16, pp. 103-114. Disponible en: https://www.revistacomunicar.com/index. php?contenido=detalles\&numero=16\&articulo=16-2001-16 [Consultado el 05/12/2017].

Lema Blanco, I.; Rodríguez Gómez, E. F. y Barranquero Carretero, A. (2016): “Jóvenes y tercer sector de medios en España: Formación en comunicación y cambios social”. Comunicar, v. 24, n. 48, pp. 91-99. Disponible en: https://doi.org/10.3916/ C48-2016-09 [Consultado el 05/12/2017]. 
Lim, M. (2012): "Clicks, cabs and coffee houses: Social media and oppositional movements in Egypt, 2004-2011". Journal of Communication, n. 62, pp. 231-248. Disponible en: https://doi.org/10.1111/j.1460-2466.2012.01628.x [Consultado el 05/12/2017].

Marí Sáez, V. M. (2016): Comunicaciones interrumpidas. Madrid: PPC.

Marquina Arenas, J. (2012): Plan social media y community manager. Barcelona: UOC.

Martín Beristain, C. y González Hidalgo, E. (2012): El oasis de la memoria. Memoria histórica y violaciones de Derechos Humanos en el Sáhara Occidental (Tomo I). Bilbao: Hegoa.

Martín Nieto, R. (2007): “La comunicación web de las ONGD”. Signo y pensamiento, v. 26, n. 51, pp. 130-136. Disponible en: http://bit.ly/1DX9q5C [Consultado el 26/02/2017].

Montoliu y Riu, L. (2012): "Las ONG en la red. Análisis de la presencia, la interactividad y la participación en Internet de las entidades del tercer sector catalán”. AE-IC. Disponible en: http://bit.ly/2qYItgl [Consultado el 13/05/2017].

ONU (2006): "Informe del Secretario General sobre la situación relativa al Sáhara Occidental, documento S/2006/249". Disponible en: http://bit.ly/2qYOqdi [Consultado el 16/02/2017].

O'Reilly, T. (2005, 30 de septiembre): “What is web 2.0". orreilly.com. Disponible en: http://goo.gl/m4Xnqm [Consultado el 06/12/2017].

Sampedro, V.; Jerez Novara, A. \& López Rey, J. (2002): “Imagen pública y estrategias de counicación”, en Blanco Revilla, M. (ed.): Las ONGs y la politica. Madrid: Istmo, pp. 251-281.

Shaw, E. (1979): "Agenda-setting and mass communication theory". Gazette International Journal for Mass Communication Studies, v. 25, n. 2, pp. 96-105.

Silva Robles, C. (2016): "Perfil del community manager en las agencias de publicidad y relaciones públicas de España". El Profesional de la Información, vol. 25, n. 2, pp. 237-245. Disponible en: https://doi.org/10.3145/epi.2016.mar.10 [Consultado el 05/12/2017].

SOGAPS (s.f.): “Página web corporativa”. Disponible en: https://sogaps.org/ [Consultado el 10/03/2017].

Soria Ibáñez, M. M. (2011): "La interacción de los públicos en las ONG 2.0: El estado actual de la comunicación social". Revista Internacional de Relaciones Públicas, vol. 1, n. 2, pp. 175-195. Disponible en: https://doi.org/10.5783/RIRP-2-201110-175-195 [Consultado el 06/12/2017].

Soria Ibáñez, M. M. (2010): "El ciberactivismo, nuevo modelo de relaciones públicas en las ONGs. Análisis del caso español". Icono 14, n. A8, pp. 288-302. Disponible en: https://doi.org/10.7195/ri14.v8i3.242 [Consultado el 05/12/2017].

Soroeta Liceras, J. (2005, 14 de noviembre): “El Sáhara treinta años después". El País. Disponible en: http://bit.ly/2qtuprE [Consultado el 23/02/2017].

Um Draiga (s.f.): "Página web corporativa". Disponible en: http://www.umdraiga.com/ [Consultado el 10/03/2017].

- (2013, 4 de abril): "Ningún país reconoce la soberanía de Marruecos sobre el Sáhara”. Um Draiga. Disponible en: http://bit.ly/2s0LrQ6 [Consultado el 23/02/2017]. 
Vidal Climent, V. (2004): “La comunicación en las organizaciones no lucrativas”, en Bel Mallén, J. I. (coord.): Comunicar para crear valor. Barañáin: EUNSA, pp. 307-322.

Vidal, P. y Creixams, C. (2006): “Existir para comunicar”. Observatorio del Tercer Sector. Disponible en: http://bit.ly/29nVVTy [Consultado el 13/05/2017].

Waterman, D.; Spence, P. R. y Van der Heide, B. (2013): "Social media as information source: Recency of updates and credibility of information”. Journal of Computer-Mediated Communication, v. 19, n. 2, pp. 171-183. Disponible en: https:// doi.org/10.1111/jcc4.12041 [Consultado el 06/12/2017]

Waters, R. D. et al. (2009): "Engaging stakeholders through social networking: How nonprofit organizations are using Facebook”. Public Relations Review, vol. 35, n. 2, pp. 102-106. Disponible en: https://doi.org/10.1016/j.pubrev.2009.01.006 [Consultado el 06/12/2017]. 